\title{
Erratum to: An exact sequence for contact- and symplectic homology
}

Frédéric Bourgeois • Alexandru Oancea

Published online: 17 March 2015

(C) Springer-Verlag Berlin Heidelberg 2015

\section{Erratum to: Invent. Math. 175, 611-680 (2009) DOI: 10.1007/s00222-008-0159-1}

The goal of this note is to make two corrections to the original article. The first correction concerns the transversality assumptions for the definition of linearized contact homology. The second correction concerns the description of the connecting map $D$ in terms of the contact complex. These corrections are described, respectively, in Sects. 1 and 2 below.

The equations in the original article are numbered by $1,2,3, \ldots$ The equations in the current note are numbered by $x .1, x .2, x .3, \ldots$ where $x$ is the number of the section.

\section{Transversality assumptions}

In this section we correct conditions $(A)$ and $\left(B_{a}\right)$ in Remark 9, p. 633 of the original article by formulating below the stronger conditions $(\widetilde{A})$ and $\left(\widetilde{B}_{a}\right)$

The online version of the original article can be found under doi:10.1007/s00222-008-0159-1.

F. Bourgeois

Laboratoire de Mathématiques d'Orsay, UMR 8628, Université Paris-Sud, Orsay, France e-mail: frederic.bourgeois@ math.u-psud.fr

A. Oancea $(\bowtie)$

Institut de Mathématiques de Jussieu-Paris Rive Gauche, Sorbonne Universités, UPMC Univ Paris 06, UMR 7586, Case 247, 4 place Jussieu, 75005 Paris, France

e-mail: alexandru.oancea@imj-prg.fr 
under which linearized contact homology is defined in a given free homotopy class $a$. As stated in Remark 9, p. 633 of the original article we expect the results of the original article to hold in complete generality once a suitable perturbation scheme has been worked out (for example the polyfold theory of Hofer et al. [5]), since our arguments are essentially independent of the details of such a scheme.

For the reader's convenience we recall the notation from the original article: $(M, \xi)$ is a contact manifold with symplectic filling $(W, \omega)$, such that $\omega$ admits a primitive near the boundary which restricts to a positive contact form $\lambda$ on the boundary; the symplectic completion of $(W, \omega)$ is denoted $(\widehat{W}, \widehat{\omega})$; $J$ is a compatible almost complex structure on $\widehat{W}$ which is cylindrical on the symplectization part of $\widehat{W} ; J_{\infty}$ is the corresponding compatible almost complex structure on the symplectization $\mathbb{R} \times M ; a$ denotes a free homotopy class of loops in $W, i: M \hookrightarrow W$ is the inclusion, and $i^{-1}(a)$ denotes the set of free homotopy classes of loops in $M$ which are mapped to $a$ via the inclusion; $\mathcal{P}_{\lambda}^{i^{-1}(a)}$ is the set of periodic Reeb orbits of $\lambda$ in $i^{-1}(a)$.

In Remark 9, p. 633 of the original article we formulated conditions $(A)$ and $\left(B_{a}\right)$ under which linearized contact homology $H C_{*}^{i^{-1}(a)}(\lambda, J)$ was supposed to be well-defined. It was brought to our attention by M. Abouzaid, J. Latschev and J. Nelson that condition $\left(B_{a}\right)$ is not strong enough as shown by the following example. Consider the ellipsoid $E:=\left\{\left(z_{1}, z_{2}\right) \in \mathbb{C}^{2}\right.$ : $\left.\left|z_{1}\right|^{2} / a_{1}^{2}+\left|z_{2}\right|^{2} / a_{2}^{2}=1\right\}$ with $0<\sqrt{2} a_{1}<a_{2}$ and $a_{1}, a_{2}$ rationally independent. The contact form is induced by $\frac{1}{2}\left(\sum x_{i} d y_{i}-y_{i} d x_{i}\right)$ on $\mathbb{R}^{4} \equiv \mathbb{C}^{2}$, and the closed Reeb orbits are iterates $\gamma_{k}^{i}, i=1,2, k \geq 1$ of the simple orbits $\gamma^{1}:=E \cap\left\{z_{2}=0\right\}, \gamma^{2}:=E \cap\left\{z_{1}=0\right\}$. Since $a_{1}, a_{2}$ are rationally independent these orbits are nondegenerate, and since $\sqrt{2} a_{1}<a_{2}$ the Conley-Zehnder indices of $\gamma_{1}^{1}, \gamma_{2}^{1}$ are $\mu\left(\gamma_{1}^{1}\right)=3, \mu\left(\gamma_{2}^{1}\right)=5$ and their grading is $\left|\gamma_{1}^{1}\right|=2,\left|\gamma_{2}^{1}\right|=4$. Let $J$ be some generic almost complex structure as above. The moduli space $\mathcal{M}\left(\gamma_{1}^{1}, \varnothing ; J\right)$ (cf. p. 630 of the original article) is nonempty and has dimension 2 (the asymptote $\gamma_{1}^{1}$ is simple and transversality can be achieved for generic $J$ ). On the other hand, the moduli space $\mathcal{M}\left(\gamma_{2}^{1}, \gamma_{1}^{1}, \gamma_{1}^{1} ; J_{\infty}\right)$ (cf. p. 629 of the original article) has virtual dimension 0 and is always nonempty, since it contains the double branched covers of the trivial cylinder over $\gamma_{1}^{1}$, which form a 2-dimensional family. Thus transversality can never be achieved for this moduli space. The point now is that pairs $[u, v] \in \mathcal{M}_{c}\left(\gamma_{2}^{1}, \gamma_{1}^{1} ; J\right)$ with $u \in \mathcal{M}\left(\gamma_{2}^{1}, \gamma_{1}^{1}, \gamma_{1}^{1} ; J_{\infty}\right)$ and $v \in \mathcal{M}\left(\gamma_{1}^{1}, \emptyset ; J\right)$ fall outside the scope of assumption $\left(B_{a}\right)$ in Remark 9, p. 633 of the original article since $\operatorname{dim} \mathcal{M}\left(\gamma_{1}^{1}, \varnothing ; J\right)>0$. On the other hand, nothing prevents $a$ priori such a pair to appear on the boundary of $\mathcal{M}\left(\gamma_{2}^{1}, \gamma_{1}^{1} ; J_{\infty}\right)$, which would destroy the relation $\partial^{2}=0$ for the differential in linearized contact homology. 
Condition $(A)$ is not sufficient either as shown by the following idealized example: an index 2 cylinder in the symplectization could break into an index 1 pair of pants and an index 1 plane in the symplectization, which can be viewed in $\widehat{W}$. In order to analyze the contribution of this configuration one needs to ensure regularity of index 1 planes in $\widehat{W}$.

Correction for conditions $(A)$ and $\left(B_{a}\right)$ of Remark 9, p. 633 of the original article

To define linearized contact homology $H C_{*}^{i^{-1}(a)}(\lambda, J)$ we must strengthen assumptions $(A)$ and $\left(B_{a}\right)$ in the original article to assumptions $(\widetilde{A})$ and $\left(\widetilde{B}_{a}\right)$ below. Thus, we assume the existence of an almost complex structure $J$ that satisfies the following two regularity conditions:

( $\widetilde{A}) J$ is regular for holomorphic planes in $\widehat{W}$ which belong to moduli spaces $\mathcal{M}\left(\gamma^{\prime}, \varnothing ; J\right)$ of virtual dimension $\leq 1$;

$\left(\widetilde{B}_{a}\right) J_{\infty}$ is regular for punctured holomorphic cylinders asymptotic at $\pm \infty$ to closed Reeb orbits in $i^{-1}(a)$, belonging to moduli spaces of virtual dimension $\leq 2$, and asymptotic at the punctures to elements $\gamma^{\prime} \in \mathcal{P}^{i^{-1}(0)}(\lambda)$ such that there exists a $J$-holomorphic building of type $0|1| k_{+}, k_{+} \geq 0$ in the sense of $[2, \S 8.1]$ with exactly one positive puncture and asymptote $\gamma^{\prime}$. (By definition, a building of type $0|1| k_{+}$has 1 level in $\widehat{W}$ and $k_{+}$levels in $\mathbb{R} \times M$.)

The main point of assumption $\left(\widetilde{B}_{a}\right)$ is that we allow the virtual dimension of the $J$-holomorphic building with asymptote $\gamma^{\prime}$ to be arbitrary, whereas in $\left(B_{a}\right)$ of the original article we had considered only buildings of virtual dimension 0 . Condition $(\widetilde{A})$ strengthens condition $(A)$ of the original article in that we also assume regularity for holomorphic planes that belong to moduli spaces of virtual dimension 1 .

We wish to stress that assumptions $(\widetilde{A})$ and $\left(\widetilde{B}_{a}\right)$ depend on $\lambda$ and $J$. In no way do they suffice to prove invariance of $H C_{*}^{i^{-1}(a)}(\lambda, J)$ with respect to deformations of $\lambda$ or $J$. As explicitly stated in Remark 7, p. 633 of the original article the invariance with respect to $\lambda$ and $J$ needs the polyfold formalism currently being developed by Hofer et al. [5]. Alternatively, invariance follows from the isomorphism between $H C_{*}^{i^{-1}(a)}(\lambda, J)$ and positive $S^{1}$-equivariant symplectic homology $S H_{*}^{S^{1}}{ }^{+}(W, \omega)$ with $\mathbb{Q}$-coefficients [4]. The proofs of the original article are written for a specific choice of $\lambda$ and $J$ that obey the assumptions above.

We give below the proof that $H C_{*}^{i^{-1}(a)}(\lambda, J)$ is defined under assumptions $(\widetilde{A})$ and $\left(\widetilde{B}_{a}\right)$. Along the way, we need to correct the Eqs. (36) and (77) in the original article. 
Correction for Eq. (36)

We denote $\mathcal{M}^{B}\left(\gamma^{\prime}, \emptyset ; J_{\infty}\right)$ the moduli space of $J_{\infty}$-holomorphic planes in the symplectization $\mathbb{R} \times M$. Its virtual dimension is $\bar{\mu}\left(\gamma^{\prime}\right)+2\left\langle c_{1}(\xi), B\right\rangle$. We define

$$
\partial_{0}: C_{*}^{i^{-1}(a)}(\lambda) \rightarrow \Lambda_{\omega}
$$

by

$$
\partial_{0}\left(\gamma^{\prime}\right):=\sum_{\substack{B \in H_{2}(M ; \mathbb{Z}) \\\left|\gamma^{\prime}\right|=\left|e^{B}\right|+1}}\left(\sum_{[F] \in \mathcal{M}^{B}\left(\gamma^{\prime}, \emptyset ; J_{\infty}\right) / \mathbb{R}} \epsilon(F)\right) e^{B} .
$$

The correct form of Eq. (36) is

$$
\partial \circ \partial=0, \quad \partial_{0}+e \circ \partial=0 .
$$

Proof of the fact that assumptions $(\widetilde{A})$ and $\left(\widetilde{B}_{a}\right)$ imply Eq. (36), namely

$$
\partial \circ \partial=0, \quad \partial_{0}+e \circ \partial=0 .
$$

We first prove the identity $\partial_{0}+e \circ \partial=0$. To prove that $\partial_{0}\left(\gamma_{1}^{\prime}\right)+e \circ \partial\left(\gamma_{1}^{\prime}\right)=0$ for $\gamma_{1}^{\prime} \in \mathcal{P}_{\lambda}^{i^{-1}(a)}$ we examine the boundary of the 1-dimensional moduli spaces $\mathcal{M}^{A_{1}}\left(\gamma_{1}^{\prime}, \emptyset ; J\right)$ for $A_{1} \in H_{2}(W ; \mathbb{Z})$ such that $\left|\gamma_{1}^{\prime}\right|-\left|e^{A_{1}}\right|=1$. By assumption $(\widetilde{A})$ the almost complex structure $J$ is regular for this moduli space, so that its boundary points come in pairs with opposite signs (this is where we use the stronger assumption $(\widetilde{A})$ instead of $(A)$ ). We claim that the boundary is given by

$$
\mathcal{M}^{A_{1}}\left(\gamma_{1}^{\prime}, \emptyset ; J_{\infty}\right) / \mathbb{R} \cup \underset{\gamma_{2}^{\prime} \in \mathcal{P}_{\lambda}}{\bigcup}\left[\mathcal{M}_{c}^{A_{2}}\left(\gamma_{1}^{\prime}, \gamma_{2}^{\prime} ; J\right) / \mathbb{R} \times \mathcal{M}^{A_{1}-A_{2}}\left(\gamma_{2}^{\prime}, \emptyset ; J\right)\right] .
$$

This implies the desired identity since the two terms in the above union correspond to $\partial_{0}\left(\gamma_{1}^{\prime}\right)$ and $e \circ \partial\left(\gamma_{1}^{\prime}\right)$ respectively (note that the sets under the second union sign in (1.1) are not necessarily disjoint). To prove that the boundary has this form we appeal to the SFT compactness theorem [2], by which the boundary elements of the above moduli space correspond to holomorphic buildings of type $0|1| k_{+}$with $k_{+} \geq 1$. It is enough to prove that $k_{+}=1$, in which case the two terms in the union correspond to the level in $\widehat{W}$ being empty, respectively non-empty. We analyze first the case in which the level in $\widehat{W}$ is non-empty. Assumption $\left(\widetilde{B}_{a}\right)$ implies that all levels in the symplectization have index $\geq 1$. Assumption $(\widetilde{A})$ implies that the components of the level in $\widehat{W}$ have index $\geq 0$ 
(this would be still true assuming only $(A)$ ). Since the total index of the building is 1 we infer that there can be a single level in the symplectization, i.e. $k_{+}=1$, and moreover that the holomorphic planes in $\widehat{W}$ by which it is capped have index 0 . This configuration corresponds to the second term in the above union. We now analyze the case in which the level in $\widehat{W}$ is empty. The same argument as before shows that $k_{+}=1$, so that the level contains a single connected component. This corresponds to the term $\mathcal{M}^{A_{1}}\left(\gamma_{1}^{\prime}, \varnothing ; J_{\infty}\right) / \mathbb{R}$ in the above union.

We now prove the identity $\partial \circ \partial=0$. To prove that $\partial \circ \partial\left(\bar{\gamma}^{\prime}\right)=0$ for $\bar{\gamma}^{\prime} \in \mathcal{P}_{\lambda}^{i^{-1}(a)}$ we examine the boundary of the 1-dimensional moduli spaces $\mathcal{M}_{c}^{A}\left(\bar{\gamma}^{\prime}, \gamma^{\prime} ; J\right) / \mathbb{R}$ for $A \in H_{2}(W ; \mathbb{Z})$ such that $\left|\bar{\gamma}^{\prime}\right|-\left|\underline{\gamma}^{\prime}\right|-\left|e^{A}\right|=2$. Assumptions $(\widetilde{A})$ and $\left(\widetilde{B}_{a}\right)$ ensure that the almost complex structure $J$ is regular for these moduli spaces, so that their boundary points come in pairs with opposite signs. We claim that the boundary is given by

$$
\begin{aligned}
& \bigcup_{\gamma_{1}^{\prime} \in \mathcal{P}_{\lambda}}\left[\mathcal{M}_{c}^{A-A_{1}}\left(\bar{\gamma}^{\prime}, \gamma_{1}^{\prime} ; J\right) / \mathbb{R} \times \mathcal{M}_{c}^{A_{1}}\left(\gamma_{1}^{\prime}, \underline{\gamma}^{\prime} ; J\right) / \mathbb{R}\right] \\
& \cup \bigcup_{\gamma_{1}^{\prime} \in \mathcal{P}_{\lambda}} \mathcal{M}_{c, 1}^{A-A_{1}}\left(\bar{\gamma}^{\prime}, \underline{\gamma}^{\prime}, \gamma_{1}^{\prime} ; J\right) / \mathbb{R}\left(\mathcal{M}^{A_{1}}\left(\gamma_{1}^{\prime}, \emptyset ; J_{\infty}\right) / \mathbb{R}\right. \\
& \left.\cup \bigcup_{\gamma_{2}^{\prime} \in \mathcal{P}_{\lambda}}\left[\mathcal{M}_{c}^{A_{2}}\left(\gamma_{1}^{\prime}, \gamma_{2}^{\prime} ; J\right) / \mathbb{R} \times \mathcal{M}^{A_{1}-A_{2}}\left(\gamma_{2}^{\prime}, \emptyset ; J\right)\right]\right) .
\end{aligned}
$$

Here $\mathcal{M}_{c, 1}^{A-A_{1}}\left(\bar{\gamma}^{\prime}, \underline{\gamma}^{\prime}, \gamma_{1}^{\prime} ; J\right)$ denotes the moduli space of punctured cylinders in the symplectization with asymptotes $\bar{\gamma}^{\prime}, \gamma^{\prime}$ capped at all but one of the punctures with rigid holomorphic planes in $\widehat{W}$, asymptotic at the special puncture to $\gamma_{1}^{\prime}$, and representing the class $A-A_{1}$ (compare with a similar moduli space in Eq. (77)). The claim implies the desired identity as follows. The signed count of the elements of the set described by the first line is the coefficient of $e^{A} \underline{\gamma}^{\prime}$ in $\partial \circ \partial\left(\bar{\gamma}^{\prime}\right)$. The signed count of the elements of the set described on the second and third line is $\sum_{\gamma_{1}^{\prime}, A_{1}} \# \mathcal{M}_{c, 1}^{A-A_{1}}\left(\bar{\gamma}^{\prime}, \underline{\gamma}^{\prime}, \gamma_{1}^{\prime} ; J\right) / \mathbb{R} \times n_{\gamma_{1}^{\prime}, A_{1}}$, where $n_{\gamma_{1}^{\prime}, A_{1}}$ describes the signed count of elements of the set described on the third line. Comparing with formula (1.1) we see that $n_{\gamma_{1}^{\prime}, A_{1}}$ is the coefficient of $e^{A_{1}}$ in $\partial_{0}+e \circ \partial\left(\gamma_{1}^{\prime}\right)$, hence equal to 0 .

To prove the claim we again appeal to the SFT compactness theorem [2], by which the boundary of the 1-dimensional moduli space $\mathcal{M}_{c}^{A}\left(\bar{\gamma}^{\prime}, \gamma^{\prime} ; J\right) / \mathbb{R}$ containing a 1-parameter family of punctured cylinders of index 2 in $\mathbb{R} \times M$ capped by rigid holomorphic planes in $\widehat{W}$ consists of holomorphic buildings of type $0|1| k_{+}$with $k_{+} \geq 2$. We prove that we have $k_{+}=2$. Indeed, assumption 
$\left(\widetilde{B}_{a}\right)$ implies that the index of all levels in the symplectization is $\geq 1$, and assumption $(\widetilde{A})$ implies that the index of all levels in $\widehat{W}$ is $\geq 0$. Since the total index is 2 we obtain that $k_{+}=2$.

The first line of (1.2) describes the situation in which $\bar{\gamma}^{\prime}, \gamma^{\prime}$ are asymptotes of punctured cylinders which lie on different levels in the symplectization. The second and third lines of (1.2) describe the situation in which $\bar{\gamma}^{\prime}, \gamma^{\prime}$ are the two asymptotes at $\pm \infty$ of a punctured cylinder of index 1 in the symplectization. This punctured cylinder is capped by rigid holomorphic planes in $\widehat{W}$ at all punctures but one, where it is capped by an index 1 holomorphic building of type $0|1| 1$. The latter is described by the third line in (1.2).

Correction for Eq. (77)

We must add in Eq. (77) the term

$$
\cup \bigcup_{\gamma_{1}^{\prime} \in \mathcal{P}_{\lambda}^{\leq \alpha}}\left[\mathcal{M}_{c, 1}^{A_{1}}\left(p^{\prime}, q, \gamma_{1}^{\prime} ; H_{\infty}^{\rho},\left\{f_{\gamma}, f_{\gamma}^{\prime}\right\}, J\right) \times \mathcal{M}^{A-A_{1}}\left(\gamma_{1}^{\prime}, \emptyset ; J_{\infty}\right) / \mathbb{R}\right]
$$

Accordingly, the first displayed equation on p. 661 must be corrected to

$$
\mathcal{M}^{A-A_{1}}\left(\gamma_{1}^{\prime}, \emptyset ; J_{\infty}\right) / \mathbb{R} \cup \underset{\gamma_{2}^{\prime} \in \mathcal{P}_{\lambda}^{\leq \alpha}}{\bigcup}\left[\mathcal{M}_{c}^{A_{2}}\left(\gamma_{1}^{\prime}, \gamma_{2}^{\prime} ; J\right) / \mathbb{R} \times \mathcal{M}^{A-A_{1}-A_{2}}\left(\gamma_{2}^{\prime}, \emptyset ; J\right)\right]
$$

and the equation $e \circ \partial\left(\gamma_{1}^{\prime}\right)=0$ on the following line has to be corrected to $\partial_{0}\left(\gamma_{1}^{\prime}\right)+e \circ \partial\left(\gamma_{1}^{\prime}\right)=0$.

Correction for the discussion of the examples in Remark 9, p. 634 of the original article

In Examples (i) and (ii) the stronger assumption $\left(\widetilde{B}_{0}\right)$ is violated, as we explain below. In Example (iii) we need to strengthen the condition $\operatorname{dim} L \geq 4$ to $\operatorname{dim} L \geq 5$, so that $(\widetilde{A})$ becomes vacuous; the rest of the discussion holds verbatim to show that $\left(\widetilde{B}_{a}\right)$ is verified.

Example (i). As in p. 633 of the original article one sees that condition $(\widetilde{A})$ is satisfied. However, assumption $\left(\widetilde{B}_{0}\right)$ is never satisfied. To see this, one can refer to the example of the ellipsoid $E=\left\{\left(z_{1}, z_{2}\right) \in \mathbb{C}^{2}:\left|z_{1}\right|^{2} / a_{1}^{2}+\left|z_{2}\right|^{2} / a_{2}^{2}=1\right\}$ with $a_{1}, a_{2}$ rationally independent and $\sqrt{2} a_{1}<a_{2}$ presented at the beginning of Sect. 1. In that example we exhibited an index 0 pair of pants in the symplectization, capped at one of the punctures by a plane of index 2 . In higher dimensions the ellipsoid $E=\left\{\left(z_{1}, \ldots, z_{n}\right) \in \mathbb{C}^{n}: \sum_{j=1}^{n}\left|z_{j}\right|^{2} / a_{j}^{2}=1\right\}$ with $a_{1}, \ldots, a_{n}$ rationally independent and $\sqrt{2} a_{1}<a_{2}<\cdots<a_{n}$ features similar properties: one can exhibit a pair of pants of negative index $-2 n+4$ capped at one of the punctures by a plane of positive index $2 n-2$. Subcritical 
Stein manifolds are obtained by attaching subcritical Weinstein handles; these always contain such contact ellipsoids which violate condition $\left(\widetilde{B}_{0}\right)$.

The paragraph that follows is conjectural: Mei-Lin Yau claims in [10] that, for a special choice of almost complex structure, the cylindrical contact homology for boundaries of subcritical Stein manifolds with vanishing first Chern class can be expressed by an explicit formula in terms of the singular homology of the filling relative to its boundary. The proof in [10] has a gap. Specifically, there is a problem in the proof of [10, Lemma7.6] which makes use of a flawed covering trick, namely the contact complex for the new contact form as $k \rightarrow \infty$ contains potentially many more generators than the ones that are considered in $[10, \S 7]$, and these new generators are not accounted for in [10, §7]. However, we believe that the main formula in [10] is correct. Then Proposition 9 is to be read as expressing the fact that a long exact sequence as the one in Theorem 1 is compatible with this computation.

Example (ii). Given an upper bound $\alpha$ on the action, we choose a Morse-Bott perturbation $\left(\widehat{\omega}_{\epsilon}, J_{\epsilon}\right)$ with $\epsilon>0$ small enough and depending on $\alpha$. While assumption $(\widetilde{A})$ is still satisfied, assumption $\left(\widetilde{B}_{0}\right)$ is not satisfied anymore. Indeed, the curves involved in condition $(\widetilde{A})$ are holomorphic planes with simple asymptote in the fibers over minima of $f$ (these have index 0 ) and holomorphic planes with simple asymptote in the fibers over the unstable manifolds of critical points of $f$ having index 1 (these have index 1). Since these holomorphic planes are regular before perturbation, they remain regular for $J_{\epsilon}$; thus $(\widetilde{A})$ is satisfied. To see that $\left(\widetilde{B}_{0}\right)$ is not satisfied, let us consider a pair of pants which doubly covers a trivial cylinder over a simple orbit in the fiber, capped at one puncture by a rigid plane in the same fiber. If the fiber lies over a critical point of $f$ of index $k$, the index of such a pair of pants is $2-k$, which is $\leq 0$ as soon as $k \geq 2$. Such a curve always exists and cannot therefore be regular.

The paragraph that follows is conjectural: once the Morse-Bott techniques in [1] will be implemented along the lines of [3] within the context of linearized contact homology, they will provide a proof of Proposition 10. Then Proposition 11 is to be read as expressing the fact that a long exact sequence as the one in Theorem 1 is compatible with the computation in Proposition 10.

Remark 1.1 Nelson [8] has recently exhibited a large class of 3-dimensional contact manifolds for which transversality can be achieved for cylindrical contact homology.

\section{The map $D$}

It was pointed out to us by T. Ekholm that Proposition 8 is true as stated only in the absence of bad orbits. In case there are bad orbits, a term is missing in 
the definition of the chain map which induces the map $D$. We describe now this additional term and complete the proof of Proposition 8.

Denote by $\mathcal{P}_{\lambda}^{b a d}$ the set of unparametrized closed Reeb orbits which are bad (see p. 627 for the definition). Given $\bar{\gamma}^{\prime}, \underline{\gamma}^{\prime} \in \mathcal{P}_{\lambda}, A \in H_{2}(W ; \mathbb{Z})$ we denote by

$$
\mathcal{M}_{2, c}^{A, \text { bad }}\left(P_{\bar{\gamma}^{\prime}}, P_{\underline{\gamma}^{\prime}} ; J\right):=\bigcup_{\gamma^{\prime} \in \mathcal{P}_{\lambda}^{\text {bad }}, A_{1} \in H_{2}(W ; \mathbb{Z})}\left[\mathcal{M}_{c}^{A_{1}}\left(\bar{\gamma}^{\prime}, \gamma^{\prime} ; J\right)\right] \times\left[\mathcal{M}_{c}^{A-A_{1}}\left(\gamma^{\prime}, \underline{\gamma^{\prime}} ; J\right)\right]
$$

the set of pairs of equivalence classes $\left(\left[u^{\prime}\right],\left[u^{\prime \prime}\right]\right)$ for the equivalence relation given by ignoring the asymptotic markers $\underline{L}^{\prime}, \bar{L}^{\prime \prime}$ corresponding to the common asymptote $\gamma^{\prime}$. The decorations " 2 " and "bad" for the moduli space are motivated by the fact that it consists of curves with two sublevels such that the intermediate asymptote is a bad orbit. In the situation $\mu\left(\bar{\gamma}^{\prime}\right)-\mu\left(\underline{\gamma}^{\prime}\right)+2\left\langle c_{1}(T W), A\right\rangle=2$ and for a generic choice of the points $P_{\gamma^{\prime}}$ the moduli spaces $\mathcal{M}_{2, c}^{A, b a d}\left(P_{\bar{\gamma}^{\prime}}, P_{\underline{\gamma}^{\prime}} ; J\right)$ are rigid and one can associate a sign $\epsilon(u)$ to each of their elements via coherent orientations and fibered products. Given an element $u \in\left[\mathcal{M}_{c}^{A_{1}}\left(\bar{\gamma}^{\prime}, \gamma^{\prime} ; J\right)\right] \times\left[\mathcal{M}_{c}^{A-A_{1}}\left(\gamma^{\prime}, \underline{\gamma^{\prime}} ; J\right)\right] \subset$ $\mathcal{M}_{2, c}^{A, b a d}\left(P_{\bar{\gamma}^{\prime}}, P_{\underline{\gamma}^{\prime}} ; J\right)$ we define a sign

$$
\bar{\epsilon}(u):=\epsilon(u) \epsilon\left(\gamma^{\prime}\right),
$$

where $\epsilon\left(\gamma^{\prime}\right) \in\{ \pm 1\}$ is uniquely determined by the relation $\delta^{0}\left(\gamma_{m}^{\prime}\right)=$ $\epsilon\left(\gamma^{\prime}\right) 2 \gamma_{M}^{\prime}$ (compare Proposition 1, p. 641 of the original article).

Given the free homotopy class $a$ in $W$ we define a map

$$
\begin{gathered}
\Delta^{\text {bad }}: C_{*}^{i^{-1}(a)}(\lambda) \rightarrow C_{*-2}^{i^{-1}(a)}(\lambda), \\
\Delta^{b a d}\left(\bar{\gamma}^{\prime}\right)=-\frac{1}{2} \cdot \sum_{\substack{\underline{\gamma}^{\prime}, A \\
\left|\underline{\gamma}^{\prime} e^{A}\right|=\left|\bar{\gamma}^{\prime}\right|-2}} \frac{1}{\kappa_{\underline{\gamma}^{\prime}}} \sum_{u \in \mathcal{M}_{2, c}^{A, b a d}\left(P_{\bar{\gamma}^{\prime}}, P_{\underline{\gamma^{\prime}}} ; J\right)} \bar{\epsilon}(u) e^{A} \underline{\gamma^{\prime}} .
\end{gathered}
$$

The correct statement of Proposition 8 is the following.

Proposition 2.1 The map $\Delta+\Delta^{\text {bad }}$ defined by (85) and (2.1) is a chain map, and induces in homology the map $D$ in the long exact sequence of Theorem 1.

As a preparation, let us denote $C M_{*}^{\text {good/bad }}$, respectively $C m_{*}^{\text {good/bad }}$ the $\Lambda_{\omega}$-submodules of the complex $B C_{*}^{i^{-1}(a)}(\lambda)$ (see p. 637 of the original article) defined by 
$C M_{*}^{\text {good } / \text { bad }}:=\bigoplus_{\gamma^{\prime} \text { good } / \text { bad }} \Lambda_{\omega}\left\langle\gamma_{M}^{\prime}\right\rangle, \quad C m_{*}^{\text {good } / \text { bad }}:=\bigoplus_{\gamma^{\prime} \text { good } / \text { bad }} \Lambda_{\omega}\left\langle\gamma_{m}^{\prime}\right\rangle$.

We denote $C M_{*}:=C M_{*}^{\text {good }} \oplus C M_{*}^{\text {bad }}$ and $C m_{*}:=C m_{*}^{\text {good }} \oplus C m_{*}^{\text {bad }}$. The components $\delta^{0}, \delta^{1}$, and $\delta^{2}$ of the differential $\delta$ behave as follows with respect to the splitting

$$
B C_{*}^{i^{-1}(a)}(\lambda)=\left(C M_{*}^{\text {good }} \oplus C m_{*}^{g o o d}\right) \oplus\left(C M_{*}^{b a d} \oplus C m_{*}^{b a d}\right)
$$

- $\delta^{0}$ vanishes on $C M_{*}^{\text {good }} \oplus C m_{*}^{\text {good }} \oplus C M_{*}^{b a d}$ and $\delta^{0}: C m_{*}^{b a d} \rightarrow C M_{*-1}^{b a d}$ is an isomorphism over $\mathbb{Q}$.

- $\delta^{1}\left(C M_{*}^{\text {bad }}\right)=0, \delta^{1}\left(C M_{*}^{\text {good }}\right) \subset C M_{*-1}, \delta^{1}\left(C m_{*}\right) \subset C m_{*-1}^{\text {good }}$.

- $\delta^{2}\left(C m_{*}\right)=0, \delta^{2}\left(C M_{*}\right) \subset C m_{*-1}$ and $\delta^{2}\left(C M_{*}^{\text {bad }}\right) \subset C m_{*-1}^{\text {good }}$.

The assertions concerning $\delta^{0}$ follow from Proposition 1 . That $\delta^{1}\left(C M_{*}^{b a d}\right)=$ 0 follows from the fact that there is an even number of choices with alternating signs for the asymptotic marker at the bad orbit with the point constraint. The same argument shows that $\delta^{1}\left(C m_{*}\right) \subset C m_{*-1}^{\text {good }}$. The last assertion on $\delta^{2}$ follows from the following computation:

$$
\begin{aligned}
\delta^{2}\left(C M_{*}^{\text {bad }}\right)=\delta^{2} \delta^{0}\left(C m_{*+1}^{\text {bad }}\right) & =-\delta^{0} \delta^{2}\left(C m_{*+1}^{\text {bad }}\right)-\left(\delta^{1}\right)^{2}\left(C m_{*+1}^{\text {bad }}\right) \\
& =-\left(\delta^{1}\right)^{2}\left(C m_{*+1}^{\text {bad }}\right) \subset C m_{*-1}^{\text {good }} .
\end{aligned}
$$

Proof of Proposition 2.1 We first reinterpret $\Delta^{\text {bad }}$ by expressing the moduli spaces $\mathcal{M}_{2, c}^{A, b a d}\left(P_{\bar{\gamma}^{\prime}}, P_{\gamma^{\prime}} ; J\right)$ in terms of moduli spaces of capped punctured $S^{1}$-parametrized holomorphic cylinders. Given $\bar{\gamma}^{\prime}, \underline{\gamma}^{\prime} \in \mathcal{P}_{\lambda}, A \in H_{2}(W ; \mathbb{Z})$ we denote

$$
\begin{aligned}
& \widetilde{\mathcal{M}}_{2, c}^{A, b a d}\left(P_{\bar{\gamma}^{\prime}}, P_{\gamma^{\prime}} ; J\right) \\
& =\bigcup \quad \widetilde{\mathcal{M}}_{c}^{A_{1}}\left(P_{\bar{\gamma}^{\prime}}, S_{\gamma}^{\prime} ; J\right) \times \widetilde{\mathcal{M}}_{c}^{A-A_{1}}\left(S_{\gamma}^{\prime}, P_{\underline{\gamma^{\prime}}} ; J\right) \text {. } \\
& \gamma^{\prime} \in \mathcal{P}_{\lambda}^{\text {bad }}, A_{1} \in H_{2}(W ; \mathbb{Z})
\end{aligned}
$$

(The moduli spaces $\widetilde{\mathcal{M}}_{c}^{A}\left(P_{\bar{\gamma}^{\prime}}, S_{\gamma}^{\prime} ; J\right)$ and $\widetilde{\mathcal{M}}_{c}^{A}\left(S_{\bar{\gamma}}^{\prime}, P_{\gamma^{\prime}} ; J\right)$ are defined on p. 670.) It follows from the definition that there is a bijective correspondence

$$
\mathcal{M}_{2, c}^{A, b a d}\left(P_{\bar{\gamma}^{\prime}}, P_{\underline{\gamma^{\prime}}} ; J\right) \sim \widetilde{\mathcal{M}}_{2, c}^{A, b a d}\left(P_{\bar{\gamma}^{\prime}}, P_{\underline{\gamma^{\prime}}} ; J\right)
$$


Hence $\widetilde{\Delta}^{b a d}:=\Theta^{-1} \circ \Delta^{b a d} \circ \Theta: C_{*}^{i^{-1}(a)}(\lambda) \otimes H_{0}\left(S^{1}\right) \rightarrow C_{*-2}^{i^{-1}(a)}(\lambda) \otimes H_{1}\left(S^{1}\right)$, where $\Theta$ is defined in (48), acts by

$$
\widetilde{\Delta}^{\text {bad }}\left(\bar{\gamma}_{M}^{\prime}\right)=-\frac{1}{2} \cdot \sum_{\substack{\underline{\gamma}^{\prime}, A \\\left|\underline{\gamma}^{\prime} e^{A \mid}\right|=\left|\bar{\gamma}^{\prime}\right|-2}} \sum_{u \in \widetilde{\mathcal{M}}_{2, c}^{A, b a d}\left(P_{\bar{\gamma}^{\prime}}, P_{\underline{\gamma}^{\prime}} ; J\right)} \bar{\epsilon}(u) e^{A} \underline{\gamma}_{m}^{\prime} .
$$

Here we use the notation $\bar{\epsilon}(u):=\epsilon(u) \epsilon\left(\gamma^{\prime}\right)$, where $\epsilon(u)$ is the coherent orientation sign for $u \in \widetilde{\mathcal{M}}_{c}^{A_{1}}\left(P_{\bar{\gamma}^{\prime}}, S_{\gamma}^{\prime} ; J\right) \times \widetilde{\mathcal{M}}_{c}^{A-A_{1}}\left(S_{\gamma}^{\prime}, P_{\underline{\gamma}^{\prime}} ; J\right) \subset$ $\widetilde{\mathcal{M}}_{2, c}^{A, b a d}\left(P_{\bar{\gamma}^{\prime}}, P_{\underline{\gamma}^{\prime}} ; J\right)$.

We denote $C_{*}^{\overline{g o o d}}=C M_{*}^{\text {good }} \oplus C m_{*}^{\text {good }}, C_{*}^{\text {bad }}=C M_{*}^{\text {bad }} \oplus C m_{*}^{\text {bad }}$ and consider the splitting $B C_{*}^{i^{-1}(a)}(\lambda)=C_{*}^{\text {good }} \oplus C_{*}^{b a d}$. With respect to this splitting, the differential $\delta$ has the form

$$
\delta=\left(\begin{array}{cc}
\tilde{\alpha} & \tilde{\beta} \\
\tilde{\gamma} & \tilde{\delta}
\end{array}\right)
$$

The map $\tilde{\delta}: C M_{*}^{b a d} \oplus C m_{*}^{b a d} \rightarrow C M_{*-1}^{b a d} \oplus C m_{*-1}^{b a d}$ is given by

$$
\tilde{\delta}=\left(\begin{array}{cc}
0 & \delta^{0} \\
0 & 0
\end{array}\right)
$$

In particular, the complex $\left(C_{*}^{b a d}, \tilde{\delta}\right)$ is acyclic over $\mathbb{Q}$. In fact, it admits the contracting homotopy $h: C_{*}^{b a d} \rightarrow C_{*+1}^{b a d}$ given by

$$
h=\left(\begin{array}{cc}
0 & 0 \\
\left(\delta^{0}\right)^{-1} & 0
\end{array}\right)
$$

By [6, Lemma 2.1.6] (Killing Contractible Subcomplexes Lemma), the map

$$
(I d,-h \circ \tilde{\gamma}):\left(C_{*}^{g o o d}, \tilde{\alpha}-\tilde{\beta} \circ h \circ \tilde{\gamma}\right) \rightarrow\left(B C_{*}^{i^{-1}(a)}(\lambda), \delta\right)
$$

is a quasi-isomorphism.

Now, the maps $\tilde{\alpha}: C M_{*}^{\text {good }} \oplus C m_{*}^{\text {good }} \rightarrow C M_{*-1}^{\text {good }} \oplus C m_{*-1}^{\text {good }}, \tilde{\beta}: C M_{*}^{\text {bad }} \oplus$ $C m_{*}^{\text {bad }} \rightarrow C M_{*-1}^{\text {good }} \oplus C m_{*-1}^{\text {good }}$ and $\tilde{\gamma}: C M_{*}^{\text {good }} \oplus C m_{*}^{\text {good }} \rightarrow C M_{*-1}^{\text {bad }} \oplus$ $C m_{*-1}^{\text {bad }}$ are given by

$$
\tilde{\alpha}=\left(\begin{array}{cc}
\pi^{\text {good }} \circ \delta^{1} & 0 \\
\pi^{\text {good }} \circ \delta^{2} & \delta^{1}
\end{array}\right), \quad \tilde{\beta}=\left(\begin{array}{cc}
0 & 0 \\
\delta^{2} & \delta^{1}
\end{array}\right), \quad \tilde{\gamma}=\left(\begin{array}{ccc}
\pi^{\text {bad }} \circ \delta^{1} & 0 \\
\pi^{\text {bad }} \circ \delta^{2} & 0
\end{array}\right),
$$


where $\pi^{\text {good }}: C_{*}^{\text {good }} \oplus C_{*}^{\text {bad }} \rightarrow C_{*}^{\text {good }}$ and $\pi^{\text {bad }}: C_{*}^{\text {good }} \oplus C_{*}^{\text {bad }} \rightarrow C_{*}^{\text {bad }}$ are the obvious projections. Note that the map $(I d,-h \circ \tilde{\gamma})$ preserves the filtration (43) and therefore induces a map of spectral sequences. This map is an isomorphism on the first page, and therefore an isomorphism on the second page as well.

Since

$$
\tilde{\alpha}-\tilde{\beta} \circ h \circ \tilde{\gamma}=\left(\begin{array}{cc}
\pi^{\text {good }} \circ \delta^{1} & 0 \\
\pi^{\text {good }} \circ \delta^{2}-\delta^{1} \circ\left(\delta^{0}\right)^{-1} \circ \pi^{\text {bad }} \circ \delta^{1} & \delta^{1}
\end{array}\right)
$$

we infer that the differential $D$ on the second page of the spectral sequence is induced by the chain map $\pi^{\text {good }} \circ \delta^{2}-\delta^{1} \circ\left(\delta^{0}\right)^{-1} \circ \pi^{\text {bad }} \circ \delta^{1}$. We have already seen in the proof of Proposition 8 that the term $\pi^{\text {good }} \circ \delta^{2}$ coincides with $\tilde{\Delta}$. That the term $-\delta^{1} \circ\left(\delta^{0}\right)^{-1} \circ \pi^{\text {bad }} \circ \delta^{1}$ coincides with $\tilde{\Delta}^{\text {bad }}$ follows directly from the definitions. This finishes the proof.

Remark 2.2 For a general filtered complex with differential $\delta=\delta^{0}+\delta^{1}+$ $\delta^{2}+\ldots$, the differential $\bar{\delta}^{2}$ on the second page of the spectral sequence is not induced by a chain map on the first page. In our situation it was possible to exhibit a chain map inducing $\bar{\delta}^{2}$ thanks to the fact that $\delta^{0}$ could be absorbed in an acyclic complex.

In the general case the definition of $\bar{\delta}^{2}$ is the following. An element $x \in$ $E_{\delta ; *, 0}^{0}$ defines a class $[x]_{1}$ in $E_{\delta ; *, 0}^{1}$ if $\delta^{0}(x)=0$ (this is automatic in our situation). This class is a cycle with respect to $\bar{\delta}^{1}$ if and only if $\delta^{1}(x)+\delta^{0}(y)=0$ for some $y \in E_{\delta ; *-1,1}^{0}$. By definition of the spectral sequence determined by a filtration (see for example [7, §2.2] and [9, Example 7.1]), the image of the corresponding class $[x]_{2} \in E_{\delta ; *, 0}^{2}$ through $\bar{\delta}^{2}$ is given by

$$
\bar{\delta}^{2}[x]_{2}:=\left[\delta^{2}(x)+\delta^{1}(y)\right]_{2} .
$$

Acknowledgments We would like to thank M. Abouzaid, T. Ekholm, J. Fish, J. Latschev, and J. Nelson for their comments on our original article. F. B.: Partially supported by ERC Starting Grant StG-239781-ContactMath. A. O.: Partially supported by ERC Starting Grant StG-259118-Stein. The present work is part of the authors activities within CAST, a Research Network Program of the European Science Foundation.

\section{References}

1. Bourgeois, F.: A Morse-Bott approach to contact homology. PhD thesis, Stanford University, Stanford (2002)

2. Bourgeois, F., Eliashberg, Y., Hofer, H., Wysocki, K., Zehnder, E.: Compactness results in symplectic field theory. Geom. Topol. 7, 799-888 (2003)

3. Bourgeois, F., Oancea, A.: Symplectic homology, autonomous Hamiltonians, and MorseBott moduli spaces. Duke Math. J. 146(1), 71-174 (2009) 
4. Bourgeois, F., Oancea, A.: $S^{1}$-equivariant symplectic homology and linearized contact homology (2012). arXiv:1212.3731

5. Hofer, H., Wysocki, K., Zehnder, E.: A general Fredholm theory. II. Implicit function theorems. Geom. Funct. Anal. 19(1), 206-293 (2009)

6. Loday, J.-L.: Cyclic homology. In: Grundlehren der Mathematischen Wissenschaften. Fundamental Principles of Mathematical Sciences, vol. 301, 2nd edn. Springer, Berlin (1998). Appendix E by María O. Ronco, Chapter 13 by the author in collaboration with Teimuraz Pirashvili

7. McCleary, J.: A user's guide to spectral sequences. In: Cambridge Studies in Advanced Mathematics, vol. 58, 2nd edn. Cambridge University Press, Cambridge (2001)

8. Nelson, J.: Applications of automatic transversality in contact homology. PhD thesis, University of Wisconsin, Madison (2013)

9. Oancea, A.: Fibered symplectic cohomology and the Leray-Serre spectral sequence. J. Symplectic Geom. 6(3), 267-351 (2008)

10. Yau, M.-L.: Cylindrical contact homology of subcritical Stein-fillable contact manifolds. Geom. Topol. 8, 1243-1280 (2004) 